\title{
Front Matter: Volume 7648
}

, "Front Matter: Volume 7648," Proc. SPIE 7648, Smart Sensor Phenomena, Technology, Networks, and Systems 2010, 764801 (21 April 2010); doi: 10.1117/12.865244

SPIE Event: SPIE Smart Structures and Materials + Nondestructive Evaluation and Health Monitoring, 2010, San Diego, California, United States 


\title{
PROCEEDINGS OF SPIE
}

\section{Smart Sensor Phenomena, Technology, Networks, and Systems 2010}

\author{
Kara J. Peters \\ Wolfgang Ecke \\ Theodore E. Matikas \\ Editors \\ 8-10 March 2010 \\ San Diego, United States \\ Sponsored by \\ SPIE \\ Cosponsored by \\ American Society of Mechanical Engineers (United States) \\ Cooperating Organizations \\ Intelligent Materials Forum (Japan) \\ Jet Propulsion Laboratory (United States) \\ National Science Foundation (United States) \\ Published by \\ SPIE
}

Volume 7648 
The papers included in this volume were part of the technical conference cited on the cover and title page. Papers were selected and subject to review by the editors and conference program committee. Some conference presentations may not be available for publication. The papers published in these proceedings reflect the work and thoughts of the authors and are published herein as submitted. The publisher is not responsible for the validity of the information or for any outcomes resulting from reliance thereon.

Please use the following format to cite material from this book:

Author(s), "Title of Paper," in Smart Sensor Phenomena, Technology, Networks, and Systems 2010, edited by Kara J. Peters, Wolfgang Ecke, Theodore E. Matikas, Proceedings of SPIE Vol. 7648 (SPIE, Bellingham, WA, 2010) Article CID Number.

ISSN 0277-786X

ISBN 9780819480637

Published by

SPIE

P.O. Box 10, Bellingham, Washington 98227-0010 USA

Telephone +1 3606763290 (Pacific Time) · Fax +1 3606471445

SPIE.org

Copyright (C) 2010, Society of Photo-Optical Instrumentation Engineers

Copying of material in this book for internal or personal use, or for the internal or personal use of specific clients, beyond the fair use provisions granted by the U.S. Copyright Law is authorized by SPIE subject to payment of copying fees. The Transactional Reporting Service base fee for this volume is $\$ 18.00$ per article (or portion thereof), which should be paid directly to the Copyright Clearance Center (CCC), 222 Rosewood Drive, Danvers, MA 01923. Payment may also be made electronically through CCC Online at copyright.com. Other copying for republication, resale, advertising or promotion, or any form of systematic or multiple reproduction of any material in this book is prohibited except with permission in writing from the publisher. The CCC fee code is 0277-786X/10/\$18.00.

Printed in the United States of America.

Publication of record for individual papers is online in the SPIE Digital Library.

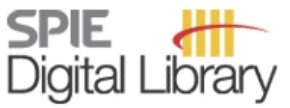

SPIEDigitalLibrary.org

Paper Numbering: Proceedings of SPIE follow an e-First publication model, with papers published first online and then in print and on CD-ROM. Papers are published as they are submitted and meet publication criteria. A unique, consistent, permanent citation identifier (CID) number is assigned to each article at the time of the first publication. Utilization of CIDs allows articles to be fully citable as soon they are published online, and connects the same identifier to all online, print, and electronic versions of the publication. SPIE uses a six-digit CID article numbering system in which:

- The first four digits correspond to the SPIE volume number.

- The last two digits indicate publication order within the volume using a Base 36 numbering system employing both numerals and letters. These two-number sets start with 00, 01, 02, 03, 04, $05,06,07,08,09,0 A, 0 B \ldots 0 Z$, followed by 10-1Z, 20-2Z, etc.

The CID number appears on each page of the manuscript. The complete citation is used on the first page, and an abbreviated version on subsequent pages. Numbers in the index correspond to the last two digits of the six-digit CID number. 


\section{Contents}

vii Conference Committee

\section{SESSION 1 FIBER BRAGG GRATING SENSORS I}

764802 Interrogation of a wavelength tunable fiber Bragg grating sensor based ring laser for dynamic strain monitoring [7648-01]

O. Balogun, Y. Zhu, S. Krishnaswamy, Northwestern Univ. (United States)

764803 High-speed full-spectrum interrogation of fiber Bragg gratings for composite impact sensing [7648-02]

S. Chadderdon, T. Vella, R. Selfridge, S. Schultz, Brigham Young Univ. (United States);

S. Webb, C. Park, K. Peters, M. Zikry, North Carolina State Univ. (United States)

764804 Impact induced damage assessment in composite laminates through embedded fiber Bragg gratings [7648-03]

S. Webb, K. Peters, M. A. Zikry, North Carolina State Univ. (United States); T. Vella,

S. Chadderdon, R. Selfridge, S. Schultz, Brigham Young Univ. (United States)

764805 Long range multiplexed sensors based on identical Bragg gratings with ultra-low reflectivity [7648-04]

M. G. Shlyagin, L. A. Arias Castro, Ctr. de Investigación Científica y de Educación Superior de Ensenada (Mexico); R. Martínez Manuel, Ctr. de Investigación Científica y de Educación Superior de Ensenada (Mexico) and Univ. of Johannesburg (South Africa); S. V. Miridonov, Ctr. de Investigación Científica y de Educación Superior de Ensenada (Mexico)

SESSION 2 FIBER BRAGG GRATING SENSORS II

764807 High-precision thermal strain measurements using surface-mounted fiber Bragg grating sensors [7648-06]

U. C. Mueller, J. Both, Technische Univ. München (Germany); J. Roths, Hochschule München (Germany); H. Baier, Technische Univ. München (Germany)

764808 Fiber optic thermal health monitoring of composites [7648-07]

M.-C. Wu, W. P. Winfree, J. P. Moore, NASA Langley Research Ctr. (United States)

764809 A film pressure sensor based on optical fiber Bragg grating [7648-09]

Z. Zhang, G. Deng, Y. Dai, Y. Liu, J. Leng, Harbin Institute of Technology (China)

\section{SESSION $3 \quad$ APPLICATIONS OF FIBER OPTIC SENSORS IN COMPOSITES}

$7648 \mathrm{OB}$ Embedded fiber Bragg sensors for damage identification in sandwich composites after impact [7648-12]

D. A. Hackney, K. J. Peters, North Carolina State Univ. (United States) 
7648 OD Multi-axial fiber-optic electric field sensor [7648-14]

D. Perry, R. Gibson, B. Schreeve, S. Schultz, D. Selfridge, Brigham Young Univ. (United States)

$7648 \mathrm{OE} \quad$ Non-contact structural damage detection using magnetic admittance approach with circuitry tuning [7648-15]

X. Wang, J. Tang, Univ. of Connecticut (United States)

7648 OF Sensitivity analysis of a smart two-directional MOSFET magnetic sensor [7648-16]

A. Abou-Elnour, Ajman Univ. of Science \& Technology (United Arab Emirates); E. Mohamed, Omm Al-Qura Univ. (Saudi Arabia); O. Abo-Elnor, Ajman Univ. of Science \& Technology (United Arab Emirates); M. Ibrahim, Ain Shams Univ. (Egypt)

\section{SESSION 5 SENSORS FOR SHM}

7648 OG A performance comparison of transducer designs for interferometric fiber optic accelerometers [7648-17]

E. A. Moro, Univ. of California, San Diego (United States) and Los Alamos National Lab. (United States); M. D. Todd, Univ. of California, San Diego (United States); A. Puckett, Los Alamos National Lab. (United States)

$7648 \mathrm{OH} \quad$ A low-cost digital image correlation based constitutive sensor [7648-18] G.-J. Yun, S. Shang, S. Kunchum, J. Carletta, The Univ. of Akron (United States); S.-B. Nam, Kangwon National Univ. (Korea, Republic of)

$76480 \mathrm{~J}$ Intelligent inventory management for packaged gases [7648-20]

A. Mason, A. Shaw, A. I. Al-Shamma'a, Liverpool John Moores Univ. (United Kingdom)

\section{SESSION 6 MICRO- AND NANO-SENSORS}

$76480 \mathrm{M}$ Self-repairing polymer optical fiber sensor [7648-23]

Y. J. Song, K. J. Peters, North Carolina State Univ. (United States)

7648 ON Embedded high resolution sensor based on optical feedback in a vertical cavity surface emitting laser [7648-24]

B. Van Hoe, D. Lamon, E. Bosman, G. Van Steenberge, J. Missinne, Univ. Gent (Belgium);

P. Goethals, Katholieke Univ. Leuven (Belgium); P. Krassimir, Vrije Univ. Brussel (Belgium);

D. Reynaerts, Katholieke Univ. Leuven (Belgium); J. Vanfleteren, P. Van Daele, Univ. Gent (Belgium)

$764800 \quad$ Polymer encapsulated 2.5D sensor arrays as building blocks for creating smart objects [7648-25]

M. Lishchynska, L. Moore, D. Rogoz, K. Delaney, J. Barrett, Cork Institute of Technology (Ireland) 


\section{SESSION 7 CIVIL INFRASTRUCTURE APPLICATIONS OF FIBER OPTIC SENSORS}

7648 OP SHM process as perceived through 350 projects (Invited Paper) [7648-26]

B. Glisic, Princeton Univ. (United States) and SMARTEC SA (Switzerland); D. Inaudi,

N. Casanova, SMARTEC SA (Switzerland)

$7648 \mathrm{OQ}$ Smart fiber-reinforced polymer anchorage system with optical fiber Bragg grating sensors [7648-28]

M. Huang, Harbin Institute of Technology (China); Z. Zhou, Harbin Institute of Technology (China), Dalian Univ. of Technology (China), and Missouri Univ. of Science and Technology (United States); J. He, Dalian Univ. of Technology (China); G. Chen, Missouri Univ. of Science and Technology (United States); J. Ou, Harbin Institute of Technology (China) and Dalian Univ. of Technology (China)

\section{SESSION 8 GUIDED WAVE SENSORS I}

7648 OS Piezoelectric wafer active sensor guided wave imaging [7648-31]

L. Yu, V. Giurgiutiu, Univ. of South Carolina (United States)

7648 OT A comparison of three optical systems for the detection of broadband ultrasound [7648-32] G. Thursby, C. McKee, B. Culshaw, Univ. of Strathclyde (United Kingdom)

7648 OU Fatigue crack detection and localization using reference-free method [7648-33]

S. Soni, S. B. Kim, A. Chattopadhyay, Arizona State Univ. (United States)

\section{SESSION 9 GUIDED WAVE SENSORS II}

7648 OV Comparison of guided wave sensors for SHM sensor networks [7648-34]

T. Windisch, B. Köhler, N. Meyendorf, Fraunhofer-Institut für Zerstörungsfreie Prüfverfahren (Germany)

7648 OW Flexible ultrasonic transducers for structural health monitoring of metals and composites [7648-35] M. Kobayashi, National Research Council Canada (Canada); K.-T. WU, J.-L. Shih, McGill Univ. (Canada); C.-K. Jen, S. E. Kruger, National Research Council Canada (Canada)

$76480 \mathrm{X}$ Subsurface material characterization using high frequency eddy current spectroscopy [7648-36] H. Hever, S. Hillmann, M. Klein, N. Meyendorf, Fraunhofer-Institut für Zerstörungsfreie Prüfverfahren (Germany)

7648 OY A physically based classification approach for identifying AE source mechanism [7648-37] D. Rajendra, A. Esterline, M. Sundaresan, North Carolina Agricultural and Technical State Univ. (United States) 
$76480 Z \quad$ Bayesian probabilistic structural modeling for optimal sensor placement in ultrasonic guided wave-based structural health monitoring [7648-38]

E. B. Flynn, M. D. Todd, Univ. of California, San Diego (United States)

764810 Machine learning approach to impact load estimation using fiber Bragg grating sensors [7648-39]

C. K. Coelho, C. Hiche, A. Chattopadhyay, Arizona State Univ. (United States)

764811 The role of embedded sensors in damage assessment in composite laminates [7648-40] C. Park, K. Peters, M. Zikry, North Carolina State Univ. (United States)

\section{POSTER SESSION}

764814 Experimental study in optical fiber temperature monitoring [7648-30] J. He, H. Dong, K. Yang, G. Zhang, Harbin Institute of Technology (China)

764815 The feasibility of low power wireless sensor devices in an industrial environment [7648-43] A. Mason, A. Shaw, A. I. Al-Shamma'a, Liverpool John Moores Univ. (United Kingdom)

764816 Study on the sensing performance of OFBG under large-scale negative strain [7648-44] C. Wang, Q. Hu, Harbin Institute of Technology (China); J. Ou, Harbin Institute of Technology (China) and Dalian Univ. of Technology (China)

764818 The application research of wireless sensor networks for SHM [7648-46] J. Wang, H. Gong, Y. Song, Xiamen Univ. (China)

764819 Dynamic forces measurement of cables in structural health monitoring [7648-47] Y. Song, J. Wang, Xiamen Univ. (China)

7648 1A A technique for autonomous structural damage detection with smart wireless sensor network [7648-48]

Y. Lei, Y. K. Mao, Y. L. Tang, J. X. Wang, Xiamen Univ. (China) 


\title{
Conference Committee
}

\author{
Symposium Chairs
}

Donald J. Leo, Virginia Polytechnic Institute and State University (United States)

Kara J. Peters, North Carolina State University (United States)

Symposium Cochairs

Norbert G. Meyendorf, Fraunhofer-Institut für Zerstörungsfreie Prüfverfahren (Germany)

Norman M. Wereley, University of Maryland, College Park (United States)

Conference Chair

Kara J. Peters, North Carolina State University (United States)

\section{Conference Cochairs}

Wolfgang Ecke, IPHT Jena (Germany)

Theodore E. Matikas, University of loannina (Greece)

\section{Program Committee}

Grigory Adamovsky, NASA Glenn Research Center (United States)

Farhad Ansari, University of Illinois at Chicago (United States)

George Y. Baaklini, NASA Glenn Research Center (United States)

Horst J. Baier, Technische Universität München (Germany)

Xiaoyi Bao, University of Ottawa (Canada)

Hartmut Bartelt, IPHT Jena (Germany)

James L. Blackshire, Air Force Research Laboratory (United States)

Rolf Brönnimann, EMPA (Switzerland)

Brian Culshaw, University of Strathclyde (United Kingdom)

Richard D. Finlayson, Physical Acoustics Corporation (United States)

Gerald U. Gerlach, Technische Universität Dresden (Germany) Joseph Grant, NASA Stennis Space Center (United States)

Wolfgang R. Habel, Bundesanstalt für Materialforschung und -prüfung (Germany)

Daniele Inaudi, Smartec S.A. (Switzerland)

Kerop D. Janoyan, Clarkson University (United States)

YeonWan Koh, FIBERPRO, Inc. (Korea, Republic of)

David A. Krohn, Light Wave Venture Consulting, LLC (United States) 
Silvio Kruger, National Research Council Canada (Canada)

Jinsong Leng, Harbin Institute of Technology (China)

Alexis Mendez, MCH Engineering LLC (United States)

Norbert G. Meyendorf, Fraunhofer-Institut für Zerstörungsfreie

Prüfverfahren (Germany)

Bernd Michel, Fraunhofer-Institut für Zuverlässigkeit und Mikrointegration (Germany)

Jeff W. Miller, Micron Optics, Inc. (United States)

Marc Nikles, Omnisens S.A. (Switzerland)

Richard H. Selfridge, Brigham Young University (United States)

Holger Speckmann, Airbus Deutschland GmbH (Germany)

Nobuo Takeda, The University of Tokyo Graduate School of Frontier Sciences (Japan)

Roderick C. Tennyson, Fiber Optic Systems Technology, Inc. (Canada)

Michael D. Todd, University of California, San Diego (United States)

Eric Udd, Columbia Gorge Research (United States)

Zhishen Wu, Ibaraki University (Japan)

Chung-Bang Yun, Korea Advanced Institute of Science and Technology (Korea, Republic of)

Zhi Zhou, Harbin Institute of Technology (China)

\section{Session Chairs}

1 Fiber Bragg Grating Sensors I

Wolfgang Ecke, IPHT Jena (Germany)

2 Fiber Bragg Grating Sensors II

Sridhar Krishnaswamy, Northwestern University (United States)

3 Applications of Fiber Optic Sensors in Composites

Kara J. Peters, North Carolina State University (United States)

$4 \quad$ Electromagnetic Sensors

Gun-Jin Yun, The University of Akron (United States)

5 Sensors for SHM

Curtis E. Banks, NASA Marshall Space Flight Center (United States)

6 Micro- and Nano-Sensors

Stephen M. Schultz, Brigham Young University (United States)

7 Civil Infrastructure Applications of Fiber Optic Sensors

Theodore E. Matikas, University of loannina (Greece)

8 Guided Wave Sensors I

Norbert G. Meyendorf, Fraunhofer-Institut für Zerstörungsfreie Prüfverfahren (Germany) 
9 Guided Wave Sensors II

Victor Giurgiutiu, University of South Carolina (United States)

10 Sensor Signal Processing and Optimization

Mannur J. Sundaresan, North Carolina Agricultural and Technical State University (United States) 
Downloaded From: https://www.spiedigitallibrary.org/conference-proceedings-of-spie on 26 Apr 2023

Terms of Use: https://www.spiedigitallibrary.org/terms-of-use 